\title{
Planktologische und hydrographisch-chemische Untersuchungen in der Eckernförder Bucht (westliche Ostsee) während und nach der Vereisung im extrem kalten Winter 1962/1963
}

\author{
W. HiCKEL \\ Btologische Anstalt Helgoland, Meeresstation, Helgoland
}

\begin{abstract}
Planctological and hydrographic-chemical investigations in the Eckernförder Bucht (western Baltic Sea) during and after the ice covering in the extreme cold winter 1962/1963. In the winter 1962/1963 the "Eckernförder Bucht" (a fjord of the western Baltic $\mathrm{Sea}$ ) and the Kieler Bucht (Kiel Bay) were ice-covered during more than two months. In this period, five sample series in $2.5 \mathrm{~m}$ intervals were taken in the outer Eckernförder Bucht beneath the ice layer (thickness 20 to $35 \mathrm{~cm}$ ); two further series were collected after the ice had disappeared. Chemical and physical measurements of water and seston properties as well as quantitative phytoplankton counts were carried out. Seston and plankton decreased during the period of ice covering. Turbidity clouds were found in medium depths. A brown coloured layer consisting of pennate diatoms was found attached to the subsurface of the ice during February and March, obviously consuming the soluble phosphate of the topmost water layer. Light measurements underneath snow-covered ice revealed that the compensation depth lays as deep as $2.5 \mathrm{~m}$ at noon. Plankton counts were very low and showed small Chaetoceras sp. and pennate diatoms to be dominant, the latter probably having detached from the ice. In the bottom water the main biomass consisted of peridinians, especially Ceratium tripos. Soon after nutrient-rich, low-salinity and highly turbid water, melted from snow and ice, appeared beneath the ice layer, a plankton bloom was found within the uppermost 1 to 2 meters on 13 th of March. This bloom consisted mainly of the diatoms Achranthes taeniata and Detonula confervacea. At this time, more favourable growth conditions were observed than after the disappearance of the ice, due to the vertical density gradient, which presumably caused a lower sinking rate, allowing the diatoms to use light and the favourable nutrient conditions beneath the ice layer. On 28th of March, three days after the ice had broken up, the diatom bloom had depleted the phosphate content at $2.5 \mathrm{~m}$ depth. The re-occurrence of wind turbulence, as well as sinking processes, distributed the phytoplankton in the now unstratified water column during the next week. Wind turbulence appeared to be the limiting factor for the now reduced increase in cell concentration within the euphotic layer. At this time, Achnantbes taeniata increased to more than $80 \%$ of the total phytoplankton biomass. This diatom previously was not reported to cause spring blooms in the western Baltic like Skeletonema costatum and several species of Chaetoceras. The zooplankton, consisting of copepods and nauplii, increased from 640 individuals per $\mathrm{m}^{3}$ on 5 th of February to $1420 / \mathrm{m}^{3}$ on 1 st of March during the ice covering, but declined during the diatom bloom to $340 / \mathrm{m}^{3}$ on 28 th of March. The significance of diatoms attached to the subsurface of the ice for primary production is discussed in the light of pertinent literature.
\end{abstract}




\section{EINLEITUNG}

Als im Winter 1962/1963 die westliche Ostsee, besonders die Kieler Bucht und die Beltsee, für Monate zufror, ergab sich die seltene Möglichkeit des Studiums biologischer, chemischer und hydrographischer Vorgänge unter der Eisdedke dieses interessanten Meeresgebiets. Unsere Untersuchungen wurden in der äußeren Eckernförder Bucht von Ende Januar bis Anfang April 1963 durchgeführt.

In der westlichen Ostsee fehlt ein regelmäßiger Gang der Vereisung. Die Art der Eisbedeckung ist sehŕ unterschiedlich; eine über Monate geschlossene Eisdecke gehört hier zu den Seltenheiten. Sie bildet sich in extremen Eiswintern, die nur $2 \%$ der Winter im Zeitraum von $1896 / 1897$ bis $1953 / 1954$ ausmachten (Atlas der Eisverhältnisse, DHI). Im extremen Eiswinter 1946/194.7 wurden z. B. in der Kieler Bucht und der Beltsee 80 bis 100 Tage mit Eisbedeckung, in der Eckernförder Bucht mehr als 80 Tage mit Eis festgestellt.

Im Winter 1962/1963 ließ starker Frost bereits über Weihnachten die inneren Förden der schleswig-holsteinischen Ostküste zufrieren. Am 8./9. Januar 1963 war Festeis auf der Eckernförder Bucht und Neueis in der Kieler Bucht. Schon am 16. Januar wurde die Eckernförder Bucht wegen einer starken Festeisdecke für die Schiffahrt geschlossen. $\mathrm{Ab}$ Mitte bis Ende Januar wurde überall in der Kieler Bucht schweres Treibeis, an den Küsten zusammenhängendes Eis und Packeisgürtel gemeldet. Am 24. Januar, nach kurzer Auflockerung am Ende des Monats dann wieder ab 7. Februar, war die ganze Kieler Bucht mit zusammenhängendem Treibeis bedeckt; die Zufahrten nach Kiel und Flensburg wurden durch Eisbrecher offengehalten. Ende Februar reichte die geschlossene Eisdecke bis östlich von Arkona.

Am 4. März 1963 war der Höhepunkt des Eiswinters. Am 6. März stellte sich die Großwetterlage um. Am 12. März setzten in der offenen Kieler Bucht Eisbewegungen ein, die stellenweise offene Rinnen, andernorts Packeiswälle entstehen ließen. Bis zum 24. März war die westliche Kieler Bucht eisbedeckt. Am 24./25. März fand mit dem Einbruch milder Meeresluft der Eisgang in der Eckernförder Bucht statt. Das Eis trieb nach Osten $a b$, während in den übrigen Förden und vor der Küste noch schweres Treibeis lag. $\mathrm{Da}$ aber das Eis bald zurückdriftete, wurden in der Eckernförder Bucht noch bis zum 8. April starke Eisgürtel beobachtet. Erst am 16. April war die Auflösung des Eises beendet; in den inneren dänischen Gewässern war das Eis erst am 24. April weitgehend verschwunden. Die Ostseeausgänge (Beltsee) vereisten früher (11. Januar) als die Kieler Bucht und wurden früher wieder frei.

In Abbildung 1 wird eine für den längsten Teil der Untersuchungsperiode typische Vereisungslage dargestellt (nach dem Eisbericht des Deutschen Hydrographischen Instituts vom 14. Februar 1963). Bei der geschlossenen Eisbedeckung westlich des Fehmarnbeltes können die Kieler Bucht und die Förden nicht mehr vom Wind beeinflußt werden. Bei etwa drei Monaten mit Eis für die untersuchte Eckernförder Bucht war der Winter 1962/1963 ein extremer Eiswinter. Nur viermal seit 1896/1897 waren Winter eisreicher. Das läßt die Bedeutung des Winters 1962/1963 für meereskundliche Untersuchungen hervortreten. Besonders für die biologischen Vorgänge im Pelagial ist eine Eisbedeckung (auch dichtes Treibeis) dadurch wichtig, daß sie die Windturbulenz ausschaltet, ferner das Lichtklima im Wasser stark beeinflußt. 


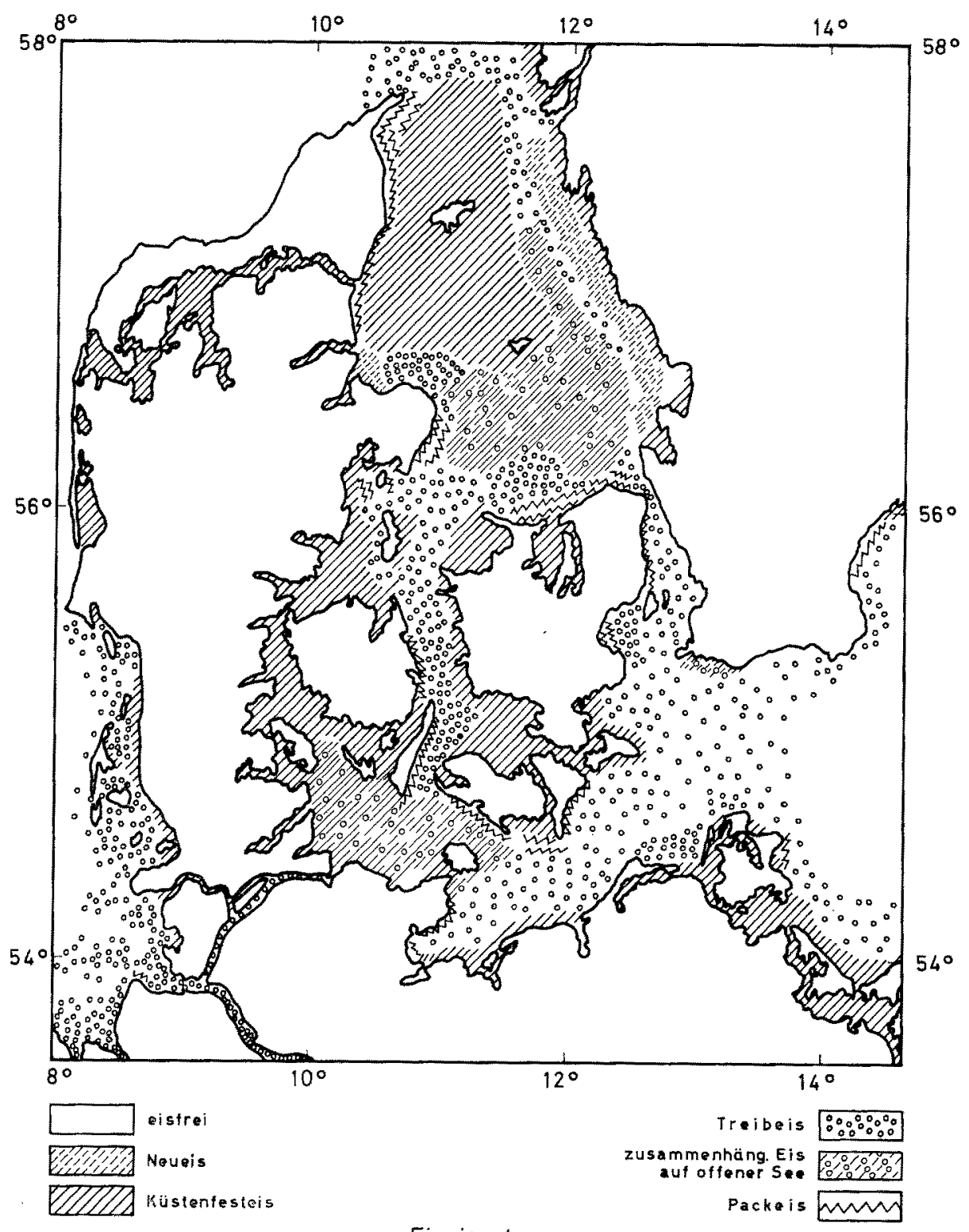

Eissignaturen

Abb. 1: Eislage in der westlichen Ostsee am 13. Februar 1963 (nach der Karte zum Eisbericht des Deutschen Hydrographischen Instituts am 14. Februar 1963, verändert). Typische Vereisungslage für den Zeitraum von Ende Januar bis Anfang März 1963 


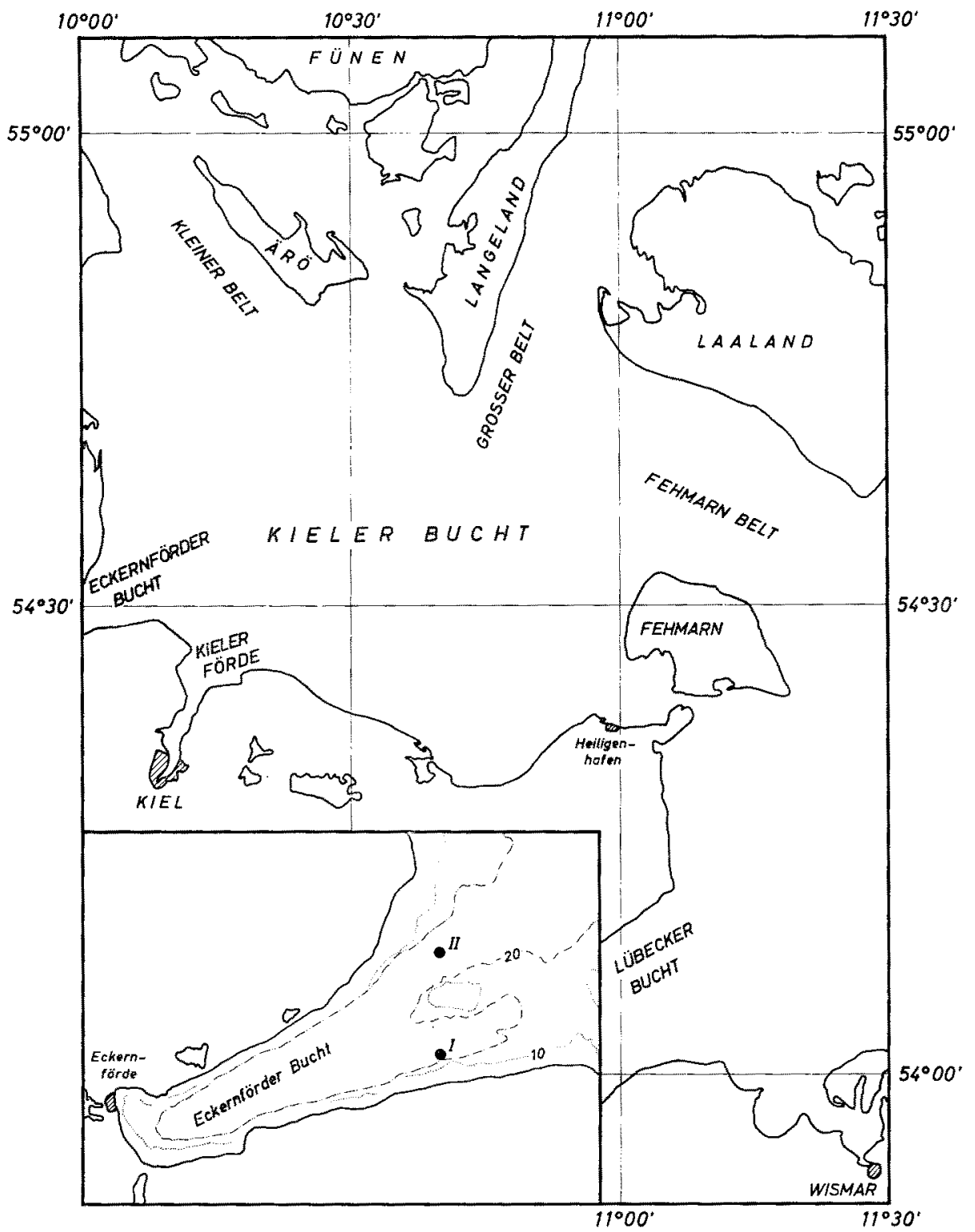

Abb. 2: Karte der Kieler Bucht und des Untersuchungsgebiets Eckernförder Bucht mit den beiden Stationen: I Eisstation ( $22 \mathrm{~m}$ Wassertiefe) und II "Boknis Eck" (28 m Tiefe), Station nach dem Eisgang 


\section{UNTERSUCHUNGSGEBIET UND PROBENNAHME}

Im Zeitraum vom 27. Januar bis 8. April 1963 wurden in der Eckernförder Bucht 7 Probenserien genommen, und zwar die ersten 5 unter dem Festeis der Förde, die letzten beiden nach dem Eisgang vom Schiff aus (Abb. 2). Die Eisstation lag $1 \mathrm{~km}$ rom Südufer der Förde nördlich von Krusendorf. Bei $22 \mathrm{~m}$ Wassertiefe wurde hier der Rand der tiefen Rinne in der Förde erreicht und damit der Bodenwasserkörper für die Probennahme zugänglich gemacht. Die letzten beiden Stationen lagen am Fördenausgang vor Boknis Eck (28 m), nördlich der Untiefe des Mittelgrundes (Abb. 2).

Die breite und tiefe Eckernförder Bucht ist zur Kieler Bucht hin weit geöffnet und entspricht deren hydrographischen Verhältnissen besonders im äußeren Teil gut (KREY et al. 1965). Uber die Hydrographie der Kieler Bucht unterrichten die Arbeiten von Wattenberg (1949), Weidemann (1948) und Kändler (1951). Die Eckernförder

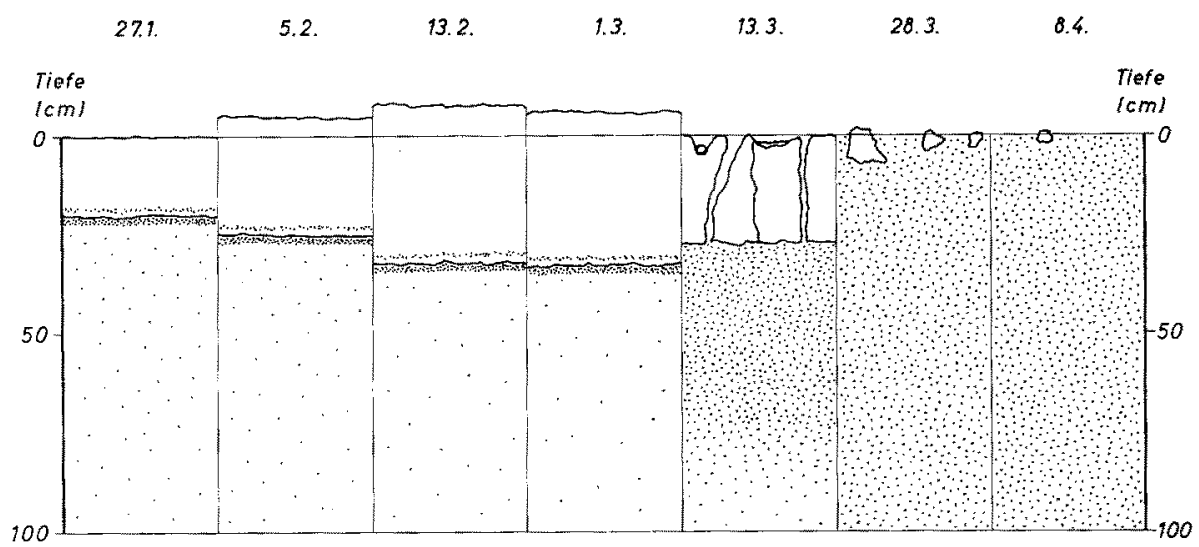

Abb. 3: Art der Eisbedeckung der Eckernförder Bucht von Ende Januar bis Anfang April 1963 und angedeutete Phytoplanktondichte im Wasser (Punktierung). Dichte Punktierung an der Eisuntergrenze und im Eis deutet festsitzende Diatomeen an

Bucht liegt außerhalb des Hauptschiffsverkehrs; nach der Schließung der Schiffahrt am 16. Januar konnte das Fördeneis überall begangen werden. Ab Mitte Januar war eine $20 \mathrm{~cm}$, später 30 bis $35 \mathrm{~cm}$ dicke Eisdecke mit Schnee vorhanden. Bei der letzten Eisbegehung am 13. März war der Schnee abgetaut und Spalten waren im Eis aufgeschmolzen (Abb. 3). Neben Sand und viel Staub wurden auf dem Eis an vielen Stellen bis faustgroße Steine und Bündel von Braunalgen (Fucus) sichtbar, die Anfang Januar am Ufer eingefroren worden waren und mit den Eisschollen verdriftet wurden. Die Eisdecke lag aber noch fest, so daß mit Sicherung durch ein auf einen Schlitten montiertes Schlauchboot und Seilsicherung das Erreichen der Station I möglich war.

Alles Gerät zur Probennahme mußte bei den ersten vier Stationen über $5 \mathrm{~m}$ hohe Packeiswälle in Ufernähe getragen werden, was die Ausrüstung begrenzte. So verbot sich die Benutzung der schweren meereskundlichen Wasserschöpfer. Durch ein Eisloch wurden 2-1-Wasserproben in 2,5 $\mathrm{m}$ Vertikalabstand mit Plastikflaschen genommen. Das 
angewandte, primitiv anmutende Prinzip der "Meyerschen Schöpfflasche" (Herausziehen eines Stopfens in der Probentiefe) funktionierte in Tiefen $>10 \mathrm{~m}$ nur mit deformierbaren Plastikflaschen, in denen sich der Innendruck dem Außendruck annähern kann. Die bei der Füllung der Flaschen aufsteigenden Luftblasen zeigen durch ihre seitliche Abdrift auch Richtung und Stärke der Wasserströmung unter dem Eis an (das Aufsteigen von Blasen unter dem Eis kann man gut hören).

Nach dem raschen Hochholen der Proben wurde sofort die Wassertemperatur (Genauigkeit $\pm 0,1^{\circ} \mathrm{C}$ ) gemessen; an abgefüllten Proben wurde der Salzgehalt bestimmt und Trübung sowie Gelbstoff nach KALLE (1956) im ELKO-II-Photometer bei 720 und $420 \mathrm{~nm}$ gemessen. Mittelwerte dreier Messungen wurden als relative Extinktionseinheiten im Vergleich zu reinstem Wasser angegeben. Das Sestongewicht wurde gravimetrisch auf $\pm 0,2 \mathrm{mg}$ ermittelt. Der Chlorophyll-a-Gehalt des Filterrückstandes wurde photometrisch nach der Extraktion mit Methanol bestimmt (Filter I 66,2, nach KREY 1939). Der Eiweißgehalt im Seston wurde mit der Biuret-Methode (KREY et al. 1957) gemessen. Phosphat-Phosphor als wichtiger Plankton-Nährstoff wurde nach WATTENBERG (1937) bestimmt.

Mit Jod-Jodkalium-Lösung („Lugolscher Lösung“) fixierte Wasserproben wurden mit der UtermöHL-Technik (UTERMöHL 1958) quantitativ auf Phytoplankton ausgezählt und nach den gebräuchlichen Schätzungen der Relation zwischen Größe und dem Kohlenstoffgehalt der Planktonorganismen auf organischen Kohlenstoff umgerechnet. Formolfixierte Netzfänge wurden zur Sicherung der Artbestimmung und zur Abschätzung der Zooplanktonmenge herangezogen. Diese Fänge wurden mit einem Netz von $56 \mu$ Maschenweite als Vertikalzüge durch die ganze Wassersäule gemacht. Bei der geringen Filtrationsleistung feiner Netze werden sie vor allem für Zooplankton nicht als quantitativ angesehen. Lichtmessungen wurden unter dem Eis mit Selen-Photoelementen vorgenommen.

\section{ERGEBNISSE UND DISKUSSION}

\section{Chemische und physikalische Eigenschaften des Sestons und Wassers}

Die Vertikalkurven der gemessenen Werte sind in den Abbildungen 4 und 5 dargestellt. Eine stärkere Temperaturschichtung war nicht ausgeprägt. Die niedrige Bodentemperatur am 5. Februar wurde durch wiederholte Messung bestätigt. Das Oberflächenwasser am 13. März zeigte eine Erwärmung, wie sie in stärkerem Maße HrLL (1967) unter dem Eis von Seen beobachtete. Eine Salzgehaltsschichtung (nicht abgebildet) wurde nur am 1. und 13. März gefunden; dabei wurde am 1. März in den obersten $5 \mathrm{~m}$ eine Salzgehaltsabnahme von 2,5\%, am 13. März eine Aussüßung durch das Schmelzwasser um etwa $3 \%$ in den obersten $2 \mathrm{~m}$ festgestellt. Ein weiterer Salzgehaltssprung von $2 \%$ lag zwischen 5 und $7,5 \mathrm{~m}$.

Der unter dem Eis sehr niedrige Sestongehalt nahm erst durch den Schmelzwasserzufluß am 13. März wieder zu. Am 28. März fiel eine Sestonanreicherung am Boden auf. An mehreren Tagen lagen zwischen 12,5 und 17,5 m Trübungs- und Gelbstoff-Maxima. 
Ob Trübungswolken durch horizontale Strömungen herbeigeführt wurden, konnte nicht untersucht werden. Es wurden aber an mehreren Untersuchungstagen unter dem Eis schwache Strömungen festgestellt, die nach Westen setzten. Die Austauschvorgänge zwischen der westlichen Ostsee und dem Kattegat gehen auch unter dem Eis weiter, sicherlich aber mit geringerer Intensität. Extrem hohe Truibungs- und Gelbstoff-Werte fielen im Schmelzwasser am 13. März auf. Es war dies der Staub, der sich in den zwei Monaten auf dem Eis angesammelt hatte. Der starken Schmelzwasser-Trübe entsprach nur eine geringe Zunahme des Sestongewichts. In einem Korrelationsdiagramm fallen diese Wertepaare daher aus dem normalen Verhältnis Trübung/Seston heraus und zeigen Partikel einer geringen Größenordnung $(<10 \mu)$ an, die eine besoncers hohe spezifische Extinktion aufweisen. Im hohen Trübungswert am 13. März in $17,5 \mathrm{~m}$ läßt sich nach der Tribung/Seston-Relation abgesunkene Schmelzwasser-Trübe vermuten.

Die Phosphatwerte nahmen am 13. Februar und 1. März von der Tiefe zur Oberfläche hin zu. Auffälliger war aber eine Phosphat-Zehrung direkt unter dem. Eis am 5. Februar bis 1. März 1963. Hier, an der Unterseite der Eisdecke, wurde ein brauner Belag festgestellt, der hauptsächlich aus pennaten "Boden"-Diatomeen bestand. Die unterste Eisschicht hatte eine ähnliche, lockere Konsistenz mit viel Wasser zwischen den Eiskristallen, wie BUNT (1963) es vom antarktischen Eis beschreibt, war aber nur etwa 0,5 bis $1 \mathrm{~cm}$ dick. Schwache, braune Streifen von Algen wurden auch im Eis beobachtet.

Offensichtlich reicht die Beleuchtung also selbst unter dem Eis für eine kräftige Photosynthese und entsprechende Nährstoffzehrung aus. Die am 5. Februar durchgeführten Lichtmessungen unter dem Eis ergaben tatsächlich eine erstaunliche Beleuchtungsstärke: die Tiefe mit 1\% des Beleuchtungswertes über dem Eis lag für den Spektralbereich maximaler Transparenz (grün) bei 2,5 $\mathrm{m}$ (Tab. 1). Dies ist allerdings der Maximalwert unter der vorhandenen Eisschicht, da mittags bei Sonne gemessen wurde. Da die 1-0/0-Tiefe als angenäherte Kompensationstiefe angesehen wird, darf eine Photosynthese der unter dem Eis festsitzenden Diatomeen nicht verwundern, zumal an der wassergetränkten Grenzschicht eine Nährstoffergänzung durch Wasseraustausch möglich ist. Die Kompensationstiefe in der benachbarten Flensburger Förde wurde im März der beiden Vorjahre mit 5 bis $15 \mathrm{~m}$ für die hellste Tagesstunde errechnet (Hrckel 1966).

Tabelle 1

Relative Beleuchtungswerte in 3 Spektralbereichen unter dem Eis der Eckernförder Bucht am 5. Februar 1963 bei Sonne, in \% vom Beleuchtungswert in Luft

\begin{tabular}{|clll|}
\hline Tiefe $(\mathrm{m})$ & Blau & Grün & Rot \\
\hline 0,85 & 1,7 & 7,5 & 2,2 \\
1,35 & 1,0 & 3,1 & 1,0 \\
1,85 & 0,7 & 2,3 & 0,3 \\
2,35 & 0,5 & 1,2 & 0,2 \\
2,85 & 0,17 & 0,9 & 0,15 \\
3,85 & & 0,85 & \\
4,85 & & 0,75 & \\
6,85 & & 0,35 & \\
12,85 & & 0,12 & \\
15,55 & & 0,0 & \\
\hline
\end{tabular}


Das Wasser unter dem Eis ist allerdings wesentlich klarer, was auch von NoRDQvisT $(1910 / 1911)$ in Seen gefunden wird.

Die Phoskatkurven zeigen ferner den enormen Nährstoffgehalt des Schmelzwassers am 13. März, der dem aufgewehten Staub zugeschrieben werden muß. Am 28. März erkennt man eine starke Phosphat-Zehrung durch die Planktonblüte, die in $2,5 \mathrm{~m}$ bereits den Nährstoff-Vorrat verbraucht hatte. In dieser Tiefe ist im März das PhotosyntheseMaximum zu erwarten (HICKEL 1967).

Nach sehr niedrigen Werten im Februar stieg mit der beginnenden Planktonblüte am 13. März auch der Chlorophyllwert an, der aber im Gegensatz zum Zählplankton bis in $5 \mathrm{~m}$ Tiefe erhöht war. Am 28. März läßt die Chlorophyllkurve neben einer hohen Phytoplanktonkonzentration in den obersten Metern ein starkes, "sekundäres" Maximum abgesunkener Zellen in $10 \mathrm{~m}$ erkennen. Am selben Tag fällt der hohe Eiweißwert im Bodenwasser auf. Der bei sehr geringer Planktonkonzentration gefundene Eiweirgehalt des Sestons dürfte auch auf Detritus und an diesem haftende Bakterien zurückgehen. Bei Eiweißwerten um Null wurden noch etwa $0,3 \mu \mathrm{g} / \mathrm{l}$ Chlorophyll gefunden.

\section{Art und Menge des Planktons}

Das Phytoplankton war unter dem Eis sehr spärlich vertreten und nahm mit der Länge der Vereisung der Kieler Bucht dauernd $a b$. Die Kurven des Kohlenstoffs im Phytoplankton (Abb. 5), wie er sich aus den Planktonzählungen errechnet, werden die kleinsten und zartesten Phytoplankter nicht quantitativ wiedergeben, da Fixierungsschäden nicht auszuschließen sind. Durch Vergleiche mit Netzfängen wurden Anzeichen für die Auflösung kleinster Chaetoceras-Zellen am 5. Februar gefunden. Am 27. Januar bestand das Phytoplankton (bis zu 40000 Zellen/1) neben Ceratium tripos aus kleinen $(10 \mu)$ pennaten Diatomeen, die besonders in $7,5 \mathrm{~m}$ und ab $20 \mathrm{~m}$ Tiefe noch häufig waren, die bei ihrer geringen Größe aber nur eine unwesentliche Biomasse darstellten. Die Häufigkeit dieser Diatomeen im Wasser war wahrscheinlich davon abhängig, wie viele dieser Zellen sich von der Eisuntergrenze, wo sie festsaßen, ablösten. Sie sanken dann im turbulenzarmen Wasser rasch ab. Ceratium tripos bildete in $2,5 \mathrm{~m}$ und am Boden den größten Teil der Biomasse.

Pennate Diatomeen dominierten im Plankton am 5. Februar 1963. Das Maximum mit 96000 Zellen/1 lag in $8 \mathrm{~m}$, ein weiteres im Bodenwasser, also abgesunkene Ansammlungen. Häufig waren auch kleine Chaetoceras-Zellen. Ceratium tripos kam in Bodennähe zahlreicher vor; Peridinium depressim und Thalassiotbrix nitzschioides wurden regelmäßig gefunden. Am 13. Februar war kaum noch Phytoplankton vorhanden, da die Diatomeen im turbulenzarmen Wasser abgesunken waren. Auch am 1. März war das Wasser bis in 17,5 $\mathrm{m}$ fast planktonfrei. Nur im Bodenwasser wurden etliche Ceratium tripos und andere große Peridineen, in 21,5 m direkt über dem Boden kleine Flagellaten $(5 \times 7 \mu)$ in der Konzentration von $64000 / 1$ angetroffen. Da diese Formen beweglich sind, darf man im Gegensatz zur Beurteilung von Diatomeenansammlungen in dieser Tiefe nicht unbedingt eine sekundäre, sondern hier eher eine primäre Anreicherung annehmen. Da für Flagellaten eine mixotrophe Ernährungsweise bekannt ist, wird eine solche Ansammlung am Boden, wo auch gelöste organische Stoffe angereichert sein werden, erklärlich. 
W. HrCKEL

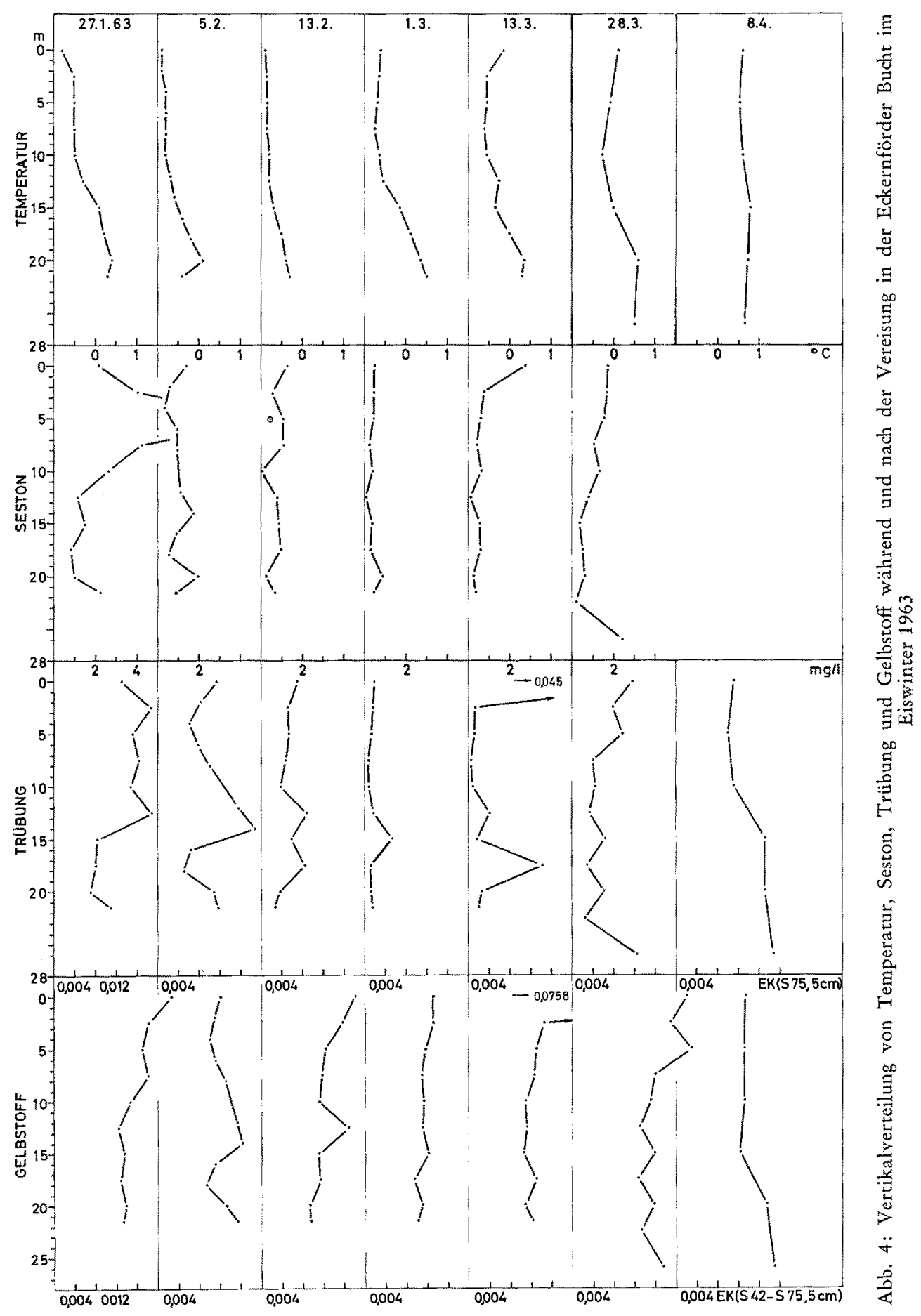




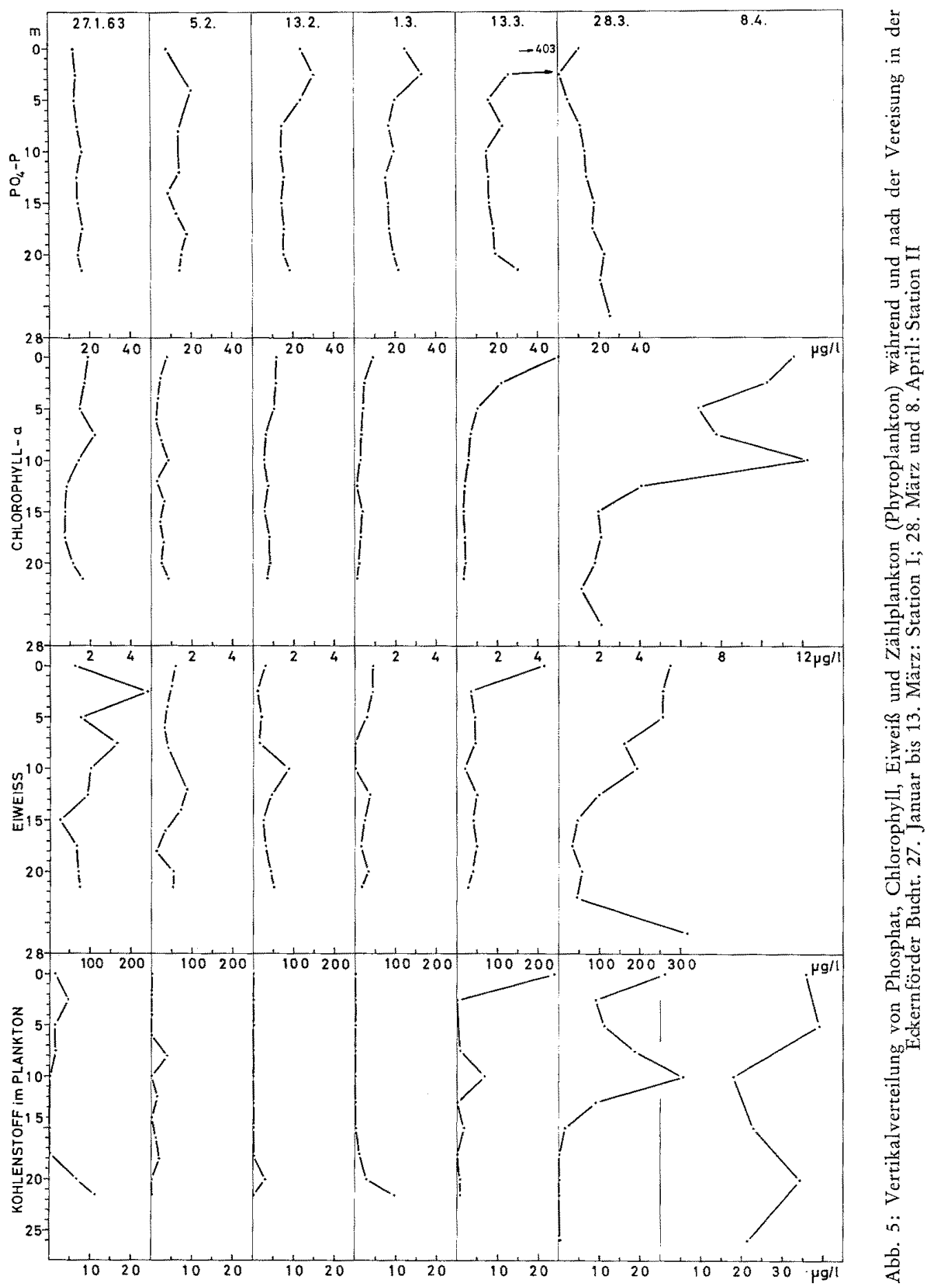


Nachdem am 13. März bereits nährstoffreiches, salzarmes Schmelzwasser unter das Eis eingesickert war, begann hier rasch eine Phytoplanktonwucherung. Die kettenbildenden Diatomeen Achnanthes taeniata und Detonula confervacea dominierten im Plankton, das sich mit mindestens 470000 Zellen/1 in der vom Schmelzwasser beeinflußten Schicht von etwa 1 bis $2 \mathrm{~m}$ Dicke ausgebreitet hatte. Außerdem waren die Diatomeengattungen Lauderia, Chaetoceras und Melosira in geringer Menge anwesend. In tieferen Schichten war nur das unbedeutende Winterplankton, besonders Ceratium tripos, anzutreffen; nur in $10 \mathrm{~m}$ wurde eine isolierte, abgesunkene Diatomeenwolke gefunden.

Am 28. März 1963, drei Tage nach dem Eisgang auf der Eckernförder Bucht, dominierte Achnanthes taeniata mit 50 bis $60 \%$ der Biomasse im Phytoplankton. Daneben spielten vor allem Detonula confervacea, weniger Coscinodiscus, Bodendiatomeen und Melosira eine Rolle. Mit Konzentrationen bis 470000 Zellen/1 hatte sich das Phytoplankton bis in $15 \mathrm{~m}$ Tiefe ausgebreitet. In $10 \mathrm{~m}$ waren abgesunkene AchnanthesZellen angereichert. Am 8. April bildete Achnanthes mit 72 bis $99 \%$, meist über $80 \%$, den weitaus größten Teil der Biomasse. Bis zu 720000 Zellen/1 wurden gezählt. Trotz starker Beleuchtung konnte die Phy toplanktonkonzentration in der euphotischen Schicht nur wenig zunehmen, da die Planktonzellen nun von der erneut wirksamen Windturbulenz in der ganzen Wassersäule verteilt wurden. Dies war deshalb so leicht möglich, weil keine haline Schichtung und damit kein vertikaler Dichtegradient vorhanden war, was sonst im Gebiet die Regel ist.

Die Diatomee Achnanthes taeniata wird sonst im Frühahrsplankton der Kieler Bucht nicht als Massenform angetroffen; sie scheint vielmehr selten zu sein. Sie erscheint beispielsweise weder in den Ostsee-Artenlisten der Plankton-Arbeiten von LoHManN (1908), noch fiel sie neuerdings bei den Frühjahrsblüten 1961 und 1962 in der nahen Flensburger Förde auf (HrCKEL 1966, 1967). Die ungewöhnlichen hydrographischen und chemischen Bedingungen im Frühjahr 1963 verhalfen hier also einer sonst produktionsbiologisch unbedeutenden Art zum ökologischen Vorteil über die häufig blütebildenden Diatomeen Skeletonema costatum und kleinen Chaetoceras-Arten.

Das Zooplankton bestand fast ausschließlich aus Copepoden und Nauplien, deren Anzahl aus Netzfängen abgeschätzt wurde. Sie nahm keineswegs wie das Phytoplankton im Laufe der Vereisung ab, sondern stieg von 640 Copepoden und Nauplien pro Kubikmeter am 5. Februar auf $1420 / \mathrm{m}^{3}$ am 1 . März 1963 an. Während der Diatomeenwucherung nahm die Anzahl der Copepoden und Nauplien von $1100 / \mathrm{m}^{3}$ am 13 . März auf $340 / \mathrm{m}^{3}$ am 28 . März ab.

\section{Abschließende Betrachtungen}

Obwohl untcr der schneebedeckten Eisschicht noch erstaunlich hohe Beleuchtungswerte gemessen wurden, konnten doch nur an der Eisunterseite festsitzende, nicht aber planktische Diatomeen, die im turbulenzarmen Wasser rasch absinken, ausreichende Strahlungsenergie für ihre Photosynthese erhalten. Leider konnten Artzusammensetzung und Zellkonzentration der Diatomeen am Eis nicht untersucht werden; dies hätte interessante Vergleiche mit den im antarktischen Eis gefundenen Diatomeen erlaubt 
(Meguro 1962, Bunt \& Wood 1963). Bunt (1963) fand bis zu 4-18 $\times 10^{6}$ Diatomeenzellen pro Liter stark wasserhaltigem Eis. Er zählte 32 Arten festsitzender, aber aud sonst planktischer Diatomeen in der Unterschicht des meterdicken antarktischen Eises im Sommer; 11 Arten davon waren häufig. Diese Population wurde als extremes Schattenplankton angesprochen. Es wurde vermutet, daß der größte Teil der Primärproduktion in den eisbedeckten Gebieten der Antarktis von diesen Algen im Eis geleistet wird. Am Eis der Eckernfördèr Bucht mögen die Zellkonzentrationen in den braun gefärbten Schichten ähnlich hoch gewesen sein, doch waren diese Schichten nur wenige Millimeter dick. Immerhin könnte auch hier ein wesentlicher Teil der Primärproduktion auf die Diatomeen am Eis zurückgehen, da die Phytoplanktonproduktion gering war.

Neben dem Plankton interessiert auch die Menge und Beschaffenheit des organischen und anorganischen Detritus, dessen schwerere Bestandteile unter dem Eis bald abgesunken waren (über Sedimentation und Abbau von Seston im Untersuchungsgebiet siehe Zetrzschel 1965). Die Auswertung von Mikrophotos des Sestons zeigte, daß nur noch Detritusflocken sowie grobe, offensichtlich vom Ufer oder aus Süßwasserzufluß stammende, pflanzliche Fasern vorkamen. Solche Fasern werden nur sehr langsam abgebaut; ihr relativer Anteil nahm mit der Länge der Vereisung zu. Biologisch ist organischer Detritus als Substrat für Bakterienansiedlung und zusammen mit diesen Bakterien als Nahrung für die Filtrierer des Zooplanktons wichtig. Man beobachtet in Nord- und Ostsee mitunter überraschend starke Copepoden-Bevölkerungen im Winter, ohne daß ihnen eine entsprechende Phytoplankton-Nahrung zur Verfügung stände. Auch bei unseren Untersuchungen wurde die größte Zooplanktonkonzentration zusammen mit einer Detritusanreicherung am 1. März 1963 gefunden.

Die günstigsten Wachstumsbedingungen für eine Wucherung der Diatomeen wurde nicht nach dem Verschwinden des Eises, sondern bei Beginn des Abschmelzens der Eisoberfläche bei noch festliegender, geschlossener Eisdecke auf der Förde festgestellt (Abbildung 3). Das salzarme Schmelzwasser, das unter das Eis floß, führte zu einer wirkungsvollen Stabilisierung der obersten Wasserschicht. Dieses Schmelzwasser war zudem sehr nährstoffreich. Schließlich dürfte das Lichtklima unter dem Eis plötzlich sehr viel besser geworden sein, als der Schnee abtaute. Die Planktonalgen konnten jetzt das günstige Licht- und Nährstoffangebot gut nutzen, weil die durch den vertikalen Dichtegradienten und noch fehlende Windturbulenz erreichte Stabilisierung der obersten 1 bis $2 \mathrm{~m}$ ein zu rasches Absinken verhinderte und die Plankter so länger in der euphotischen Zone exponiert waren.

Mit den Wachstumsbedingungen des Phytoplanktons ab Mitte März 1963 lag ein „Modellfall" für die ökologischen Voraussetzungen einer Frühjahrsblüte vor. Gerade die auffällig an den abschmelzenden Eisrand in nordischen Meeren gebundene Frühjahrsblüte hat ja zum Verständnis der Voraussetzungen für eine plötzliche Massenentwicklung des Phytoplanktons gefüht (Gran 1929, Marshall 1958). Der Einfluß der Windturbulenz erwies sich immer wieder als der ökologisch wichtigste Faktor in windreichen Meeresgebieten, sogar in der halin geschichteten Ostsee (HICKEL 1967). Bei einer Stabilisierung der obersten Meter z. B. in Nord- und Ostsee könnte selbst im Winter eine Phytoplanktonwucherung erwartet werden. Dazu läßt es aber der Wind im $\mathrm{Zu}$ sammenhang mit einer geringen Kompensationstiefe selten kommen. 


\section{ZUSAMMENFASSUNG}

1. Im extremen Eiswinter 1962/1963 waren die Edkernförder Bucht und die ganze Kieler Bucht über 2 Monate eisbedeckt. In der äußeren Eckernförder Bucht wurden ab Ende Januar 1963 fünfmal Probenserien in 2,5 $\mathrm{m}$ Vertikalabstand unter der 20 bis $35 \mathrm{~cm}$ dicken Eisdecke genommen und zweimal nach dem Eisgang wiederholt. Chemische und physikalische Messungen der Wasser- und Sestoneigenschaften sowie quantitative Phytoplanktonzählungen wurden durchgeführt.

2. Seston- und Phytoplanktongehalt des Wassers nahmen nach dem Beginn der Vereisung dauernd ab. Trübungswolken wurden in mittleren Tiefen beobachtet. An der Unterseite der Eisdecke wurde im Februar und März eine braune Schicht festsitzender, pennater Diatomeen entdeckt, deren Phosphat-Zehrung eine lebhafte Photosynthese vermuten ließ. Beleuchtungsmessungen unter dem Eis ergaben eine Kompensationstiefe von $2,5 \mathrm{~m}$ zur Mittagszeit. Das wenige Plankton bestand bis zum 5. Februar aus kleinen Chaetoceras-Arten und pennaten Diatomeen, die sich wahrscheinlich vom Eis abgelöst hatten und bei den späteren Proben das Phytoplankton zahlenmäßig beherrschten. Große Peridineen, besonders Ceratium tripos, bildeten meist den Hauptanteil der Biomasse vor allem im Bodenwasser, wo sie häufiger waren.

3. Als am 13. März der Schnee auf dem Eis geschmolzen war und sehr nährstoffreiches, salzarmes und stark getrubtes Schmelzwasser unter das Eis sickerte, begann rasch eine Planktonblüte in den obersten 1 bis $2 \mathrm{~m}$. Daran waren ganz überwiegend die Diatomeen Achnanthes taeniata und Detonula confervacea beteiligt. Es herrschten jetzt günstigere Wachstumsbedingungen als später nach dem Verschwinden des Eises, da der Zufluß des Schmelzwassers eine Stabilisierung der obersten Meter der Wassersäule durch Ausbildung eines vertikalen Dichtegradienten bewirkte. Das Plankton wurde dadurch am raschen Absinken gehindext und länger in der euphotischen Zone exponiert, so daß es das Licht- und Nährstoffangebot gut nutzen konnte.

4. Am 28. März, nach dem Eisgang, hatte die Diatomeenblüte den Phosphatvorrat in $2,5 \mathrm{~m}$ bereits verbraucht. Die nun wieder wirksame Windturbulenz verteilte zusammen mit Absinkvorgängen bis zum 8. April das Plankton bei fehlender haliner Schichtung in der ganzen Wassersäule und stellte den begrenzenden Faktor für den geringen Zuwachs der Zellkonzentration in der euphotischen Schicht dar. Der Anteil von Achnanthes taeniata nahm bis meist über $80 \%$ der Biomasse zu. Diese Diatomee wird sonst nicht als Massenform im Frühjahrsplankton der westlichen Ostsee beobachtet. Sie erhielt durch die besonderen hydrographisch-chemischen Bedingungen nach der Vereisung offenbar einen ökologischen Vorteil gegenüber den sonst blütebildenden Diatomeen wie Skeletonema und Chaetoceras sp.

5. Das Zooplankton bestand fast ausschließlich aus Copepoden und Nauplien, deren Anzahl während der Vereisung von $640 / \mathrm{m}^{3}$ am 5. Februar auf mindestens $1420 / \mathrm{m}^{3}$ am 1. März anstieg, während der Phytoplanktonentfaltung aber auf $340 / \mathrm{m}^{3}$ am 28 . März abnahm.

6. Die Bedeutung der an der Unterseite der Eisdecke festsitzenden Diatomeen für die Primärproduktion der eisbedeckten Eckernförder Bucht wird durch Vergleich mit Literaturangaben über Algen im Polareis diskutiert. 
Danksagungen. Herrn Dr. B. Zertzscher wird an dieser Stelle herzlich für seine Hilfe bei den Eisstationen gedankt, ebenso Frau U. NelleN, Frau Dr. B. Hickel und Frau B. ZeITZschel. Ohne ihren Einsatz wären die Untersuchungen nicht möglich gewesen. Frl. U. WRIEDT fühtte die Planktonzählungen durch, Herr Dr. G. Dreses half bei der Diatomeen-Bestimmung. Herrn Dr. J. LeNz verdanke ich werrvolle Hinweise und Kritik des Manuskripts. Frl. U. Volz und Herrn K. Treutner sei für ihre Mitarbeit gedankt.

\section{ZITIERTE LITERATUR}

A tLas der Eisverhältnisse der Deutschen Bucht und westlichen Ostsee, 1956. Deutsches Hydrographisches Institut, Hamburg.

BunT, J. S., 1963. Diatoms of antarctic sea-ice as agents of primary production. Natkre, Lond. $199,1255-1257$.

- \& Wood, E. J. F., 1963. Microalgae and antarctic sea-ice. Nature, Lond. 199, 1254-1255.

GraN, H. H., 1929. Investigations of the production of plankton outside the Romdalsfjord 1926/27. Rapp. P.-v. Réun. Cons. perm. int. Explor. Mer 56, 1-112.

Hrckel, W., 1966. Untersuchungen über die Phytoplanktonblüte in der Beltsee (chemische und biologische Messungen des Frühjahrs- und Herbstplanktons beim Feuerschiff „Flensburg"). Kiel, Math.-nat. Diss., 123 pp.

- 1967. Untersuchungen über die Phytoplanktonblüte in der westlichen Ostsee. Helgoländer wiss. Meeresunters. 16, 3-66.

HILL, H., 1967. A note on temperatures and water conditions beneath lake ice in spring. Limnol. Oceanogr. 12, 550-552.

Eisbericht, 1962/63. Amtsblatt des Deurschen Hydrographischen Instituts, Hamburg.

KäNDLER, R., 1951. Der Einfluß der Wetterlage auf die Salzgehaitsschichtung im Übergangsgebiet zwischen Nord- und Ostsee. Dt. hydrogr. Z. 4, 150-160.

Kalle, K., 1956. Chemisch-hydrographische Untersuchungen in der inneren Deutschen Budht. Dt. bydrogr. Z. 9, 55-65.

Krey, J., 1939. Die Bestimmung des Chlorophylls in Meerwasser-Schöpfproben. J. Cons. perm. int. Explor. Mer 14, 201-209.

- Banse, K. \& Hagmeter, E., 1957. Uber die Bestimmung von Eiweiß im Plankton mittels der Biuret-Reaktion. Kieler Meeresforsch. 13, 35-40.

- Koske, P. H. \& Szekielda, K. H., 1965. Produktionsbiologische und hydrographische Untersuchungen in der Edkernförder Bucht. Kieler Meeresforsch. 21, 135-143.

LohmanN, H., 1908. Untersuchungen zur Feststellung des vollständigen Gehaltes des Meeres an Plankton. Wiss. Meeresunters. (Abt. Kiel) 10, 129-370.

Marshall, P. T., 1958. Primary production in the Arctic. J. Cons. perm. int. Explor. Mer 23, 173-177.

Meguro, H., 1962. Plankton ice in the Antarctic Ocean. Antarctic Rec. 14, 72-79.

Nordevist, O., 1910/11. Uber das Eindringen des Lichtes in von Eis und Schnee bedeckten Seen. Int. Revue ges. Hydrobiol. Hydrogr. 3, 79-83.

UTERMöHL, H., 1958. Zur Vervollkommnung der quantitativen Phytoplanktonmethodik. Mitt. int. Verein. theor, angew. Limnol. 9, 1-38.

Wattenberg, H., 1937. Methoden zur Bestimmung von Phosphat, Silikat, Nitrit, Nitrat und Ammoniak im Seewasser. Rapp. P.-v. Réun. Cons. perm. int. Mer 103, 5-26.

- 1949. Salzgehaltsverteilung in der Kieler Bucht und ihre Abhängigkeit von Strom- und Wetterlage. Kieler Meeresforsch. 6, 17-30.

WeIDEMANN, H., 1948. Uber periodische und unperiodische Erscheinungen im Wasseraustausch der Beltsee. Kiel, Math.-nat. Diss., 43 pp. nebst Anh.

Zeitzschel, B., 1965. Zur Sedimentation von Seston, eine produktionsbiologische Untersuchung von Sinkstoffen und Sedimenten der westlichen und mittleren Ostsee. Kieler Meeresforsch. 21, 55-80.

Anschrift des Autors: Dr. W. Hrckex

Biologische Anstalt Helgoland, Meeresstation

2192 Helgoland 